\title{
Strengths and Weaknesses of Semi-Structured Interviews in Qualitative Research: A Critical Essay
}

Charles Kakilla ${ }^{1}$

\begin{abstract}
A semi-structured interview (SSI) is one of the essential tools in conduction qualitative research. This essay draws upon the pros and cons of applying semi-structured interviews (SSI) in the qualitative research method. Moreover, the challenges of SSI during the coronavirus pandemic are critically discussed to provide plausible recommendations.
\end{abstract}

\section{Introduction}

Multiple studied models portray semi-structured interviews (SSI) in critical qualitative research as indispensable tools to uncover knowledge through interaction, conversations, and subjects from different life experiences. Moreover, the shared stories and life experience about other matters are negotiably interpreted to expand the knowledge into multiple platforms.

While researchers can shape the practical knowledge of conducting SSI, it is vital to adhere to academically approved standards that yield high-quality data development. Additionally, diverse pros and cons are appreciated in undertaking SSI, and therefore this part of the paper has discussed the depth of these strengths and weaknesses.

\section{Strengths of semi-structured interviews}

The SSI are practical for undertaking in-depth conversation. Usually, the researcher can critically scrutinise the conversations and varied initially superficial responses during the SSI to arrive at multilayered conclusions. A researcher can further follow up most of the times, all verbal and non-verbal responses, such as hunches, laugher and silence, to reveal hidden information that may turn out to be helpful in the final data analysis of different themes extracted from the conversation (Ritchie \& Lewis, 2003).

The interviewers can synthesise different themes to bring about flexibility. A researcher can discuss various topics with multiple themes in SSI. Moreover, this interactive nature of the

\footnotetext{
${ }^{1}$ Corresponding author: Trinity Centre for Global Health, Trinity College Dublin, Dublin Ireland kakillac@tcd.ie ORCID: https://orcid.org/0000-0001-9837-6564
} 
SSI provides room for free responses from the interviewee. Additionally, other researchers suggested that the development of computer-assisted qualitative data analysis using flexible coding, if used on well-coded themes, can yield reliable analysis from large (more than 30) interview samples (Deterding \& Waters, 2018).

The generative nature of the SSI produces a greater likelihood to inspire new ideas to be put into play. Regarding flexible coding, an in-depth interview, as described above, Deterding and Waters (2018) argued that:

Applying thematic codes to reduced data during validation and refinement increases the likelihood that the coding is reliable and valid across hundreds or thousands of pages of data. In short, the process allows researchers to spend more time developing and elaborating concepts - and meaningfully linking them to data - than on cleaning up and combining line-by-line codes (p. 26).

Facts on topics during the SSI are captured in their natural forms. A well presented SSI can ideally draw upon the interviewee's inner voice when active participation from both parties (the interviewer and the interviewee) is underway. In a study conducted to quantify human mobility to enhance infections disease models, the SSI method from 160 people revealed about 1,161 more locations of visited places compared to tracking data from global positioning system enabled devices (Paz-Soldan et al., 2014). The author added that "the semi-structured interviews provided richer context and were easier to process...it was the only option for identifying locations retrospectively" (Paz-Soldan et al., 2014, p. 2).

\section{Weaknesses of semi-structured interviews}

Data loss could potentially occur when conducting SSI due to the following reasons; first, SSI is not feasible when they are not conducted face-to-face (e.g., online platforms such as video conferencing tools, emails, and similar settings) or written interviews. Second, limited probing due to interviewing people with language barriers (Marshall \& While, 1994). Hence, therefore, the interview can be affected by the use of technology since acquiring data in their natural form would be hugely jeopardised. Although given the present coronavirus pandemic and how it has affected physical gatherings and travel, researchers are susceptible to rely on the use of technology for critical qualitative research, particularly for SSI, along with other methods. On the other hand, the language barrier issue can be sorted by employing translators, which further pose a risk to loss of direct meaning through translation. The loss in translation 
is also discussed in details on poor or limited understanding and responses between interviewee and interviewer.

Limited understanding of the topic when the conversation is not flexible during SSIs would often kill the conversation. On the negative side, having many non-responders could alternatively result in the population's underrepresentation needed to be studied (Denzin, 2017). It's also shown that some interviewees could respond less to 'obvious' topics or subjects that are the interviewees too ashamed to speak about (Nguyen, 2015).

Poor or limited responses would kill the conversation. To a greater extent, the SSI would result in sub-standard quality whenever the interviewer loses track of thought or attempt to extrapolate the unintended meaning from a dull conversation. Small (2008), in his seminal report Lost In Translation, emphasised that; "in qualitative research, if methods are languages, then the most important issue facing qualitative researchers - especially those concerned about the science of their work - is translation". Sometimes the limited response could be caused by a language barrier, resulting in loss of data in translation and often issues in reliability and validity of the interview data (Louise Barriball \& While, 1994).

Interestingly, sometimes limited responses from interlocutors could result due to distinct cultural values. For example, according to western culture, gazing someone directly into their eyes while talking/listen to them could denote paying attention to a conversation. But the same gesture could mean being disrespectful in Eastern or African culture, especially when a younger person gazes into an older person. A compelling study by Nguyen (2015) in Vietnam showed that during SSI, some common gestures such as looking someone directly into their eyes could stir away a chance of building a good relationship with the interviewee. Again Vietnamese study participants were more prone to converse using non-verbal gestures, such as nodding heads. Or they would be friendly if recruited in the face-to-face encounter rather than having an online interview. If considered, cultural values are essential in building a good relationship with study participants before the SSI for a better revealing conversation.

Attempting to carry out an interview schedule in SSI sometimes requires third party approval if high profile individuals are the researcher's study subjects, hence inconveniences in setting up the interview. Therefore the interviewees (in this case, the high-status individuals) might need to see the questions before the actual meeting.

As active listening is an essential skill to a quality SSI, it is not ideal to conduct grouped SSI. Interviewing numerous people could alternatively employ skills from other methods such as structured interviews or focused group discussions. 
Difficulty in replicating actual recognition and acknowledgement upon emotional response. After a compassionate and emotional response, a researcher noted that:

\begin{abstract}
Often, the recollection and telling of the interview takes place long after the event was originally felt. We [critical qualitative researchers] are left with the question of how to understand the meaning of a per- son's experience with this impossibility of replicating for oneself the feelings of the participant. The best we can do is to retell the person's story while telling our own story as researcher (Nunkoosing, 2005, p. $6)$.
\end{abstract}

Frequently, scenarios like described above are implausible to occur against a backdrop of none face-to-face interviews.

\title{
Conclusions
}

Generally, the SSIs are highly appreciated in their power to engage in deep conversation, flexibility, and generative nature, hence stimulating new ideas. Additionally, the views are hauled in their natural forms, including non-verbal communication. The weaknesses of SSI include; data loss either by use of technology that come closer to imitating the conditions of face-to-face conversations or limited probing due to language barrier; loss in translation; little understanding or inadequate response to the topic. If considered, cultural values are essential in building a good relationship with study participants before including them in SSI for a better revealing conversation. Besides, SSI may also lead to time inconveniences for highprofile interviewee; SSI not ideal for grouped interviews as it requires active listening.

The current global pandemic urges the need to expand new, and possibly better, ways of carrying out SSI without potential loss of data. Therefore, the contemporary observable advances in science and technology could introduce revolutionary methods in critical qualitative research.

\section{Bibliography}

Denzin, N. K. (2017). The Research Act: A Theoretical Introduction to Sociological Methods. Routledge. https://doi.org/10.4324/9781315134543

Deterding, N. M., \& Waters, M. C. (2018). Flexible Coding of In-depth Interviews: A Twenty-first-century Approach. Sociological Methods and Research. https://doi.org/10.1177/0049124118799377

Louise Barriball, K., \& While, A. (1994). Collecting data using a semi-structured interview: a discussion paper. Journal of Advanced Nursing, 19(2), 328-335. https://doi.org/10.1111/j.1365-2648.1994.tb01088.x

Marshall, S. L., \& While, A. E. (1994). Interviewing respondents who have English as a second language: challenges encountered and suggestions for other researchers. Journal of Advanced Nursing, 19(3), 566- 


\section{1. https://doi.org/10.1111/j.1365-2648.1994.tb01122.x}

Nguyen, T. Q. T. (2015). Conducting semi-structured interviews with the vietnamese. Qualitative Research Journal, 15(1), 35-46. https://doi.org/10.1108/QRJ-04-2014-0012

Nunkoosing, K. (2005). The problems with interviews. Qualitative Health Research, 15(5), 698-706. https://doi.org/10.1177/1049732304273903

Paz-Soldan, V. A., Reiner, R. C., Morrison, A. C., Stoddard, S. T., Kitron, U., Scott, T. W., ... VazquezProkopec, G. M. (2014). Strengths and Weaknesses of Global Positioning System (GPS) Data-Loggers and Semi-structured Interviews for Capturing Fine-scale Human Mobility: Findings from Iquitos, Peru. PLoS Neglected Tropical Diseases, 8(6), e2888. https://doi.org/10.1371/journal.pntd.0002888

Ritchie, J., \& Lewis, J. (2003). Qualitative research practice: A guide for social science students and researchers. SAGE.

Seidman, I. (2006). Interviewing as qualitative research: A guide for researchers in education and the social sciences. Teachers college press.

Small, M. L. (2008). Lost In Translation: How Not to Make Qualitative Research More Scientific. Report from workshop on interdisciplinary standards for systematic qualitative research. Washington. DC. 